\title{
Estimativa da gordura corporal através de equipamentos de bioimpedância, dobras cutâneas e pesagem hidrostática
}

\author{
Maurício Nunes Rodrigues ${ }^{1}$, Sidney Cavalcante da Silva ${ }^{2}$, \\ Walace David Monteiro ${ }^{2-3}$ e Paulo de Tarso Veras Farinatti ${ }^{1}$
}

\section{RESUMO}

A estimativa do percentual de gordura $(\% \mathrm{G})$ pela bioimpedância (BIA) tem como vantagem a simplicidade da medida. Contudo, a confiabilidade da BIA tem sofrido críticas. O objetivo deste estudo foi comparar a estimativa do $\% \mathrm{G}$ através das técnicas de bioimpedância (RJL-101; Byodinamics A-310, Maltron BF-900 e BF-906), de dobras cutâneas (DC) e da pesagem hidrostática (PH). Observaram-se 25 indivíduos, homogeneizados segundo raça (branca), gênero (masculino) e idade (18 a 36 anos). Para a medida de BIA foi utilizada a padronização proposta por Lukaski et al. $(1985,1986)$. Para as DC foram utilizadas as equações de $\sum 3$ DC e $\sum 7$ DC (Jackson, Pollock, 1978). Os valores de $\% \mathrm{G}$ e de volume residual para PH foram preditos, respectivamente, pelas equações de Siri (1961) e Goldman e Becklake (1959). A análise estatística compreendeu: a) comparação entre os métodos através da ANOVA com medidas repetidas seguida de testes post-hoc de Tukey; b) correlação de Pearson $(r)$; e c) cálculo do erro padrão de estimativa (SEE) das técnicas em relação à PH. Os resultados indicaram que: a) As medidas de BIA não diferiram significativamente, entre si, para o \%G estimado; b) As medidas dos aparelhos A-310 e BF-906 não coincidiram com a PH ( $\mathrm{p}<0,01)$; c) Em geral, os valores de SEE apresentados pela BIA foram altos; d) Os valores de $r$ oscilaram entre 0,35 (RJL-101) e 0,70 (BF-906); e) As técnicas de DC apresentaram correlações maiores e SEE menores com a PH,

1. Laboratório de Atividade Física e Promoção da Saúde - UERJ.

2. Laboratório de Fisiologia, Nuicaf - Aeronáutica.

3. Programa de Pós-Graduação em Educação Física - UGF, Rio de Janeiro, RJ.

Recebido em: 21/6/2001.

Aceito em: 15/7/2001.

Endereço para correspondência:

Maurício Nunes Rodrigues

Av. Vítor Konder, 409/101

22611-250 - Rio de Janeiro, RJ, Brasil

E-mail: rodriguesm@ig.com.br

Rev Bras Med Esporte - Vol. 7, № 4 - J ul/ Ago, 2001 quando comparados com os da BIA. Apesar dos resultados, não há dados que permitam indicar um aparelho em detrimento de outros. Os resultados da BIA equivaleram-se quanto à estimativa do \%G, mas a técnica de DC mostra-se tão ou mais confiável para tanto. Contudo, os resultados devem ser ratificados pela ampliação da amostra e controle de maior número de variáveis intervenientes.

Palavras-chave: Composição corporal. Bioimpedância. Pesagem hidrostática. Dobras cutâneas. Medida e avaliação.

\section{ABSTRACT}

Comparison of body fat estimation by bioelectric impedance, skinfold thickness, and underwater weighing

The main advantage of the bioelectric impedance method (BIA) in the determination of body fat $(\% B F)$ is the simplicity of the procedure. However, its accuracy and reliability have been criticized. The purpose of this study was to compare the \%BF obtained by BIA (RJL-101; Biodynamics A-310, Maltron BF-900 e BF-906), by skinfold thickness (ST), and by underwater weighing (UW). Twenty-five subjects, divided in homogenous groups according to age (18 to 36 years), sex (men), and race (white) participated in the study. BIA measures were taken using the Lukaski et al. standardization $(1985,1986)$. ST was taken by using the equation of 3 and 7 skinfolds (Jackson, Pollock, 1978). The values of \%BF and residual volume for the UW were estimated by the Siri (1961) and Goldman and Becklake (1959) equations. Statistical analysis was calculated by: a) repeated measures ANOVA followed by the Tukey post-hoc test; b) Pearson (r) correlation; c) standard error of estimate (SEE) of the BIA and ST compared to UW. The results indicated that: a) there were no significant differences for $\% B F$ measures obtained by the BIA devices; $b$ ) the \%BF obtained by the A-310 and BF-906 devices did not match with the UW measures ( $p<0.01)$; $c$ ) SEE was high for all devices, except for the RJL-101; d) the correlation coefficients were low to moderate for all devices, the BF-906 showing the higher values; e) The ST showed greater val- 
ues of $\mathrm{r}$ and fewer SEE than the BIA. In conclusion, the findings do not allow to state that a BIA device is better than the others to assess \%BF, but the ST seems to be more powerful and reliable than the BIA for \%BF estimation. Notwithstanding, the results should be confirmed by more sophisticated experimental designs, with a closer control of sampling bias for type I and II errors.

Key words: Body composition. Bioelectric impedance. Underwater weighing. Skinfold thickness. Testing.

\section{INTRODUÇÃO}

A determinação dos componentes da composição corporal possui diversas aplicações em programas direcionados à promoção da saúde e treinamento físico-desportivo. Heyward e Stolarczyk ${ }^{1}$ apontam as seguintes possibilidades: a) identificação do risco de saúde associado com níveis excessivamente altos ou baixos da gordura corporal total; b) identificação do risco de saúde associado com o acúmulo excessivo de gordura intra-abdominal; c) monitorização de possíveis alterações da composição corporal, associadas a certas doenças; d) acompanhamento do crescimento, desenvolvimento, maturação e alterações da composição corporal relacionadas à idade; e) formulação de recomendações dietéticas e prescrição de exercício e avaliação da efetividade das mesmas. $\mathrm{O}$ acúmulo excessivo de gordura para determinada massa corporal é, reconhecidamente, um fator de risco para diversas condições patológicas, como o diabetes, a hipertensão e a doença coronariana ${ }^{2}$.

Entre as técnicas mais utilizadas na determinação dos componentes da composição corporal destacam-se as dobras cutâneas e a utilização de índices relacionando a massa corporal à estatura, cada uma com suas vantagens e limitações. Como métodos menos utilizados, podem-se mencionar a pesagem hidrostática e a tomografia computadorizada, cujas medidas investem-se de grande precisão. No entanto, são de difícil execução e custo elevado, sendo utilizadas normalmente em ambiente laboratorial ${ }^{3,4}$. Com o avanço da tecnologia, observa-se tendência para o desenvolvimento de técnicas de estimativa da composição corporal mais sofisticadas para o uso fora do ambiente laboratorial. Uma delas, objeto de crescente número de estudos, é a técnica da bioimpedância. Sua utilização, com finalidade de determinar o fracionamento da composição corporal, vem-se popularizando nas últimas décadas. Contudo, os resultados obtidos em muitos trabalhos revelam-se, freqüentemente, discrepantes ${ }^{5-12}$. Silva ${ }^{11}$ cita, ainda, diversos estudos que obtiveram correlações entre o percentual de gordura medido por bioimpedância e densitometria não maiores que 0,80 . Dentre as possíveis razões para essa variabilidade poderiam ser mencionadas diferenças metodo- lógicas quanto às populações analisadas, aos aparelhos utilizados e metodologias diferentes de coleta de dados ${ }^{11,13,14}$. Dessa forma, pode-se afirmar que pairam, no mínimo, muitas dúvidas quanto à confiabilidade e à precisão da técnica da bioimpedância, quando comparada com outros recursos.

Torna-se, então, necessário número maior de pesquisas com o objetivo de testar os diferentes aparelhos comercializados e a especificidade de suas equações preditivas para diferentes populações, a fim de obter evidências acerca da validade, precisão e confiabilidade das medidas. O presente estudo insere-se nesse contexto. Pretende-se comparar alguns dos aparelhos de bioimpedância existentes $(A-310$ da Biodynamics Corporation, BF-900 e BF-906 da Maltron e o modelo 101 da $R J L I n c$.), no que diz respeito às várias informações que fornecem e, mais precisamente, o percentual de gordura, com técnicas reconhecidamente válidas para determinação da composição corporal - a técnica de dobras cutâneas e, principalmente, a pesagem hidrostática.

\section{MATERIAL E MÉTODOS}

\section{Amostragem}

Foi analisada uma amostra não-probabilística intencional, formada por 25 voluntários do sexo masculino, brancos, com idade entre 19 e 36 anos $(24 \pm 5$ anos [média \pm dp]), em sua maioria universitários e militares, recrutados entre os alunos da Universidade do Estado do Rio de Janeiro (UERJ) e da Universidade da Força Aérea (Unifa). O nível de atividade física dos participantes poderia ser classificado de sedentário a moderado (praticantes de atividades físicas, em média, por três vezes por semana). Todos os sujeitos assinaram termo de consentimento pós-informado, conforme estabelecido pela convenção de Helsinki.

\section{Material}

Para a determinação da massa corporal e estatura dos indivíduos foi utilizada uma balança (Filizola, mod. 31, Brasil) com graduação de $100 \mathrm{~g}$ e um estadiômetro portátil (Ghrum Polar Manufacture, Suíça) com precisão de 1,0mm, respectivamente. Para a tomada dos dados de bioimpedância foram utilizados os seguintes aparelhos: A-310 (Biodynamics Corporation, EUA), BF-900 e BF-906 (Maltron, Reino Unido) e o modelo 101 (RJL Inc., EUA) com eletrodos de gel para ECG (Lectec Corporation, EUA). As medidas de dobras cutâneas foram obtidas com a utilização de um compasso (Lange, EUA) com leitura de $0,5 \mathrm{~mm}$. A coleta dos dados foi realizada em ficha própria, sendo tabulada em uma planilha de cálculo elaborada pelo autor, utilizando o software MS-Excel (Microsoft, EUA). A pesa- 
gem hidrostática foi realizada em tanque próprio, localizado no Núcleo do Instituto de Ciências da Atividade Física (Nuicaf) da Aeronáutica. Para a determinação da massa corporal submersa foi utilizada uma balança (Kratus, Brasil), com precisão de $5 \mathrm{~g}$.

\section{Métodos}

Todas as medidas foram realizadas em um mesmo dia, na parte da manhã. Os indivíduos respeitavam um período de jejum de dez horas antes da tomada das medidas, tendose abstido de atividades físicas e de bebidas alcoólicas, respectivamente, nas 12 e 24 horas precedentes. Antes das medidas, eram explicados os procedimentos necessários à coleta de dados, sendo entregue a cada voluntário o termo de consentimento para que assinassem, liberando a sua participação no estudo.

Inicialmente, eram aferidas as medidas de estatura e massa corporal e a obtenção de dez dobras cutâneas, segundo a metodologia proposta por Pollock e Wilmore ${ }^{2}$, nos seguintes sítios: peitoral, biciptal, triciptal, subescapular, axilar média, supra-ilíaca, supra-espinhal, abdominal, coxa e perna. Para o cálculo da estimativa da densidade corporal foram utilizadas as equações do somatório de três e de sete dobras desenvolvidas por Jackson e Pollock ${ }^{15} \mathrm{e}$, para o percentual de gordura, a de Siri ${ }^{16}$. Em seguida, foi realizada a estimativa da gordura percentual com a utilização dos aparelhos de bioimpedância em disposição tetrapolar, conforme proposta por Hoffer et al. ${ }^{17} \mathrm{e}$ modificada por Lukaski et al. ${ }^{5,6}$, na ordem determinada pelo método do quadrado latino, sendo registrados os valores de gordura percentual, impedância e reatância corporais, quando fornecidos pelo aparelho.

As medidas de bioimpedância eram executadas uma vez com cada aparelho, sem a retirada dos eletrodos dos pontos de contato, com o indivíduo deitado, em decúbito dorsal, em uma maca, sem portar relógio ou qualquer outro objeto metálico ${ }^{1,13,14}$. Antes da colocação dos eletrodos na pele dos sujeitos, era feita limpeza dos pontos de contato com algodão embebido em álcool. Os indivíduos ficavam repousando durante cinco minutos antes das tomadas de medidas. Para o cálculo da gordura percentual a partir do aparelho da marca $R J L$ foi utilizada a equação fornecida pelo fabricante para a estimativa da massa isenta de gordura: $\mathrm{FFM}=\left\{0,5 \times\left[\mathrm{S}^{1,48} / \mathrm{Z}^{0,55}\right]\right\} / 1,21+0,42 \times \mathrm{MCT}+0,49$, em que $\mathrm{S}$ é a estatura em $\mathrm{cm}, \mathrm{Z}$ corresponde à impedância e MCT é a massa corporal do indivíduo em quilos. Os aparelhos da Maltron e da Byodinamics forneciam o percentual de gordura diretamente, através de equações já programadas pelos fabricantes nos próprios instrumentos. Deve ser relatado um pequeno problema de alimentação observado no aparelho da $R J L$, o que impossibilitou a medida da totalidade da amostra, justificando o $n$ mais reduzido apresentado nesse instrumento.

Por fim, foi realizada a mensuração da densidade corporal pela técnica da pesagem hidrostática. Antes da tomada das medidas foram passadas aos indivíduos as instruções para a realização das mesmas, citadas por Pollock e Wilmore $^{2}$. Para a determinação da massa corporal submersa optamos por adaptar o critério desenvolvido por Behnke e Wilmore ${ }^{18}$. Foram feitas de oito a 12 tomadas de medida e considerava-se a média entre as três maiores, com variação de no máximo 5g, como a medida final. Acreditamos que, com esse critério, obtém-se uma medida final mais homogênea em relação às diversas tomadas. $\mathrm{O}$ volume residual foi calculado segundo a equação de Goldman e Becklake $^{19}$, recomendada por Pollock e Wilmore ${ }^{2}$, em que o $\mathrm{VR}=0,017$ (idade) $+0,027$ (estatura em $\mathrm{cm})-3,477$, sendo posteriormente utilizada na fórmula para a determinação da densidade corporal ${ }^{20}$ para posterior determinação do percentual de gordura através da equação de Siri ${ }^{16}$.

\section{Tratamento estatístico}

A comparação entre os métodos deu-se através de técnicas de análise de variância (ANOVA) para medidas repetidas, seguida, quando necessário, do teste post-hoc de Tukey (software Statistica, Statsoft Co., 1999). Aplicou-se, então, o índice de correlação de Pearson $(r)$ com o objetivo de analisar a associação da medida entre cada aparelho/ técnica e a pesagem hidrostática, e o cálculo do erro padrão de estimativa (SEE), em cada um dos aparelhos de bioimpedância e na técnica de dobras cutâneas, como indicativo da dispersão. Para esses cálculos, tomaram-se como critério de comparação os resultados da pesagem hidrostática. Observamos certa discordância na literatura consultada sobre que equação utilizar para o cálculo do erro padrão de estimativa ${ }^{9,21-23}$. Utilizamos a seguinte formula: $\mathrm{SEE}=$ Dp $\left(1-r^{2}\right)^{1 / 2}$ proposta por Jackson et al. ${ }^{9} \mathrm{e}$, também, por Graves et al., ${ }^{22} \mathrm{em}$ estudos de validação da BIA. Foi adotado como critério de significância estatística nível de 5\% para o erro do tipo I.

\section{RESULTADOS E DISCUSSÃO}

Inicialmente, com o objetivo de caracterizar a amostra estudada, a tabela 1 apresenta os valores da estatística descritiva para algumas variáveis antropométricas.

Como se pode observar, a amostra foi composta por 25 indivíduos, homogeneizados segundo a raça (branca), gênero (masculino) e idade (18 a 36 anos), representando adultos jovens. Com essa homogeneização procurou-se minimizar a influência de variáveis intervenientes no resultado obtido. Na tabela 2, apresentam-se os resultados obtidos pelos quatro aparelhos de bioimpedância, pelo somatório 
de três e sete dobras cutâneas e pela técnica de pesagem hidrostática, utilizada neste estudo como padrão de critério para a variável medida, ou seja, o percentual de gordura. Os valores encontram-se expressos em gordura percentual.

Realizou-se ANOVA para medidas repetidas entre os dados obtidos pelos aparelhos de bioimpedância, assim como entre estes e as medidas obtidas pelo somatório das dobras cutâneas e pela pesagem hidrostática. Os resultados indicaram haver diferenças significativas para as estimativas de $\% \mathrm{G}$ fornecidas, tanto no grupo composto por todos os equipamentos $(n=14, p<0,001)$, quanto no grupo no qual excluímos o aparelho da $R J L(\mathrm{n}=25, \mathrm{p}<0,001)$. Posteriormente, aplicou-se o teste post-hoc de Tukey e obtiveramse os resultados apresentados na tabela 3.

\section{TABELA 1}

Características da amostra - estatística descritiva

\begin{tabular}{lcccc}
\hline \multicolumn{1}{c}{ Variáveis } & Média & $\begin{array}{c}\text { Desvio } \\
\text { padrão }\end{array}$ & Máximo & Mínimo \\
& & & & \\
Idade (anos) & 24 & 5 & 36 & 19 \\
Estatura (cm) & 175,1 & 5,2 & 184,1 & 163,9 \\
Massa corporal (kg) & 73,7 & 8,3 & 86,3 & 57,8 \\
IMC $\left(\mathrm{kg} / \mathrm{m}^{2}\right)$ & 24,1 & 2,7 & 29,0 & 19,9 \\
\hline
\end{tabular}

A seguir, calculou-se o coeficiente de correlação de Pearson $(r)$ para poder-se analisar o grau de associação entre os resultados de cada aparelho, da técnica de dobras cutâneas e da técnica da pesagem hidrostática. Igualmente, determinou-se o erro padrão de estimativa, tomando por base as medidas da pesagem hidrostática. Os resultados podem ser observados na tabela 4.

Antes de iniciar a discussão dos resultados obtidos, seria importante frisar as limitações metodológicas inerentes ao presente estudo. Inicialmente, é evidente que o $n$ amostral reduzido aumenta os riscos de os resultados não refle-

\section{TABELA 2}

Estatística descritiva para o \% obtido pelos diferentes aparelhos de BIA, pela técnica de dobras cutâneas ( $\sum$ 3DC e $\sum$ 7DC) e pesagem hidrostática (PH)

\begin{tabular}{lcccr} 
Equipamento & Média & $\begin{array}{c}\text { Desvio } \\
\text { padrão }\end{array}$ & Máximo & Mínimo \\
A-310 $(\mathrm{n}=25)$ & 19,4 & 5,6 & 33,4 & 10,9 \\
$B F-900(\mathrm{n}=25)$ & 17,4 & 7,1 & 30,1 & 6,3 \\
$B F-906(\mathrm{n}=25)$ & 18,4 & 6,3 & 31,1 & 10,2 \\
$R J L-101(\mathrm{n}=14)$ & 19,1 & 3,8 & 26,8 & 13,1 \\
$\sum 3 D C(\mathrm{n}=25)$ & 12,0 & 5,7 & 26,6 & 2,6 \\
$\sum$ 7DC $(\mathrm{n}=25)$ & 12,5 & 5,7 & 25,3 & 3,0 \\
PH $(\mathrm{n}=25)$ & 14,7 & 6,2 & 25,4 & 4,8 \\
\hline
\end{tabular}

TABELA 3

Resultado da ANOVA para medidas repetidas seguida de testes post-hoc de Tukey entre técnicas de BIA, de dobras cutâneas ( $\sum$ 3DC e $\sum$ 7DC) e pesagem hidrostática (PH)

\begin{tabular}{|c|c|c|c|c|c|c|c|}
\hline Aparelhos/técnicas & $A-310$ & $B F-900$ & $B F-906$ & $R J L-101$ & $\sum 3 \mathrm{DC}$ & $\sum 7 \mathrm{DC}$ & $\mathrm{PH}$ \\
\hline$A-310(\mathrm{n}=25)$ & - & & & & & & \\
\hline$B F-900(\mathrm{n}=25)$ & NS & - & & & & & \\
\hline$B F-906(\mathrm{n}=25)$ & NS & NS & - & & & & \\
\hline$R J L-101(\mathrm{n}=14)$ & NS & NS & NS & - & & & \\
\hline$\sum 3 \mathrm{DC}(\mathrm{n}=25)$ & $p<0,01$ & $p<0,01$ & $\mathrm{p}<0,01$ & $\mathrm{p}<0,05$ & - & & \\
\hline$\sum 7 \mathrm{DC}(\mathrm{n}=25)$ & $p<0,01$ & $p<0,01$ & $p<0,01$ & $p<0,05$ & NS & - & \\
\hline $\mathrm{PH}(\mathrm{n}=25)$ & $p<0,01$ & NS & $p<0,01$ & NS & NS & NS & - \\
\hline
\end{tabular}

NS: diferença não-significativa

TABELA 4

Resultados da correlação produto-momento de Pearson $(r)$ e erro padrão de estimativa (SEE) do percentual de gordura para os aparelhos de bioimpedância e para a técnica de dobras cutâneas em relação à $\mathrm{PH}$

\begin{tabular}{|c|c|c|c|c|c|c|}
\hline $\begin{array}{l}\text { Aparelhos/ } \\
\text { técnicas }\end{array}$ & $\begin{array}{c}A-310 \\
\text { (n = 25) }\end{array}$ & $\begin{array}{l}B F-900 \\
(\mathrm{n}=25)\end{array}$ & $\begin{array}{l}B F-906 \\
(\mathrm{n}=25)\end{array}$ & $\begin{array}{l}R J L-101 \\
\text { (n = 14) }\end{array}$ & $\begin{array}{c}\sum 3 \mathrm{DC} \\
(\mathrm{n}=25)\end{array}$ & $\begin{array}{l}\sum 7 \mathrm{DC} \\
(\mathrm{n}=25)\end{array}$ \\
\hline$r$ & $0,36 \mathrm{NS}$ & $0,55 p<0,01$ & $0,70 p<0,01$ & $0,35 \mathrm{NS}$ & $0,85 p<0,001$ & $0,84 p<0,001$ \\
\hline SEE & 5,2 & 5,9 & 4,5 & 3,6 & 3,0 & 3,1 \\
\hline
\end{tabular}


tirem o comportamento da população. Também importante foi a impossibilidade de medir diretamente o volume residual para a estimativa do ar presente nos pulmões durante a técnica da pesagem hidrostática. Embora esse ponto não seja um consenso na literatura ${ }^{2,21,24}$, os erros associados a essa limitação podem levar a distorções da ordem de 500 a $1.000 \mathrm{ml}$ na estimativa do volume residual, finalizando em erros de até 2,0\% a 5,5\% na estimativa da gordura corporal ${ }^{2}$. Igualmente digno de nota é o fato de que não se pôde garantir que o mecanismo de calibragem dos equipamentos de bioimpedância estivesse funcionando a contento, assim como a não realização da medida da água corporal total de cada indivíduo, para análise comparativa com os dados obtidos pelos aparelhos. Acredita-se, porém, que o impacto dessas limitações sobre os resultados, ao menos no âmbito da amostra testada, não seja grande a ponto de invalidar as observações.

Utilizou-se o aparelho RJL-101 como padrão de medida da técnica de bioimpedância, por este ser largamente empregado na literatura, tanto na validação da técnica propriamente dita como no desenvolvimento de equações preditivas para os componentes da composição corporal por essa técnica, e pelo reduzido número de trabalhos envolvendo os outros aparelhos. Fica evidenciado que os resultados indicaram não haver diferenças nas estimativas de \% G fornecidas entre os aparelhos de bioimpedância, como se pode observar pela ausência de diferenças significativas apresentadas nas comparações entre os instrumentos (tabela 3). No entanto, quando se comparam os resultados obtidos com os da pesagem hidrostática e com os estimados pelas dobras cutâneas, percebem-se discrepâncias.

Lohman ${ }^{25}$ desenvolveu um padrão, baseado empiricamente em erros de medida associados à pesagem hidrostática, para avaliar os erros de predição de equações para estimativa tanto do percentual de gordura, quanto da massa isenta de gordura. De acordo com o padrão proposto, o erro padrão de estimativa para o percentual de gordura deveria variar até 2,0\% para ser considerado ideal; até 2,5\% para ser classificado como excelente; até 3,0\% para ser muito bom; até 3,5\% seria classificado como bom; até 4,0\% para ser considerado regular; até $4,5 \%$ considerando-o fraco e, finalmente, até $5,0 \%$ sendo classificado como não recomendado. Podem-se, então, comparar os dados obtidos neste estudo com a análise de outros envolvendo o fracionamento dos constituintes da composição corporal.

Os resultados do teste de Tukey (tabela 3) revelaram diferenças significativas em relação à pesagem hidrostática em dois aparelhos: o modelo A-310 da Byodinamics e o Maltron BF-906. No A-310, além de suas medidas não coincidirem satisfatoriamente com as da $\mathrm{PH}$, o valor de $r$ alcançado (tabela 4) indica que suas variações não obedece-

Rev Bras Med Esporte - Vol. 7, № 4 - J ul/ A go, 2001 ram a um mesmo padrão, apresentando ainda alto valor do erro padrão de estimativa, indicando variação de medida em relação à PH muito alta. Já o BF-906, embora seus resultados apresentassem diferenças significativas em comparação com a $\mathrm{PH}$, indicando carência em sua precisão, suas medidas variaram na mesma proporção, apresentando a correlação mais significativa do estudo, ainda que moderada. O erro padrão de estimativa apresentado por esse aparelho, embora menor do que os dos modelos $B F-900$ e $A$ 310 , ainda se encontra um pouco distante do padrão considerado bom, uma vez aceitos os padrões sugeridos por Lohman ${ }^{25}$.

Dentre os aparelhos testados, aquele que demonstrou os maiores índices de concordância e associação com a pesagem hidrostática foi o Maltron BF-900, mesmo apresentando o maior índice de erro padrão de estimativa e correlação não muito forte, mas com elevados níveis de significância ( $\mathrm{p}<0,01)$. Igualmente, a estimativa de gordura através do aparelho RJL-101 não apresentou diferenças significativas com a pesagem hidrostática. Como já mencionado, o RJL-101 foi utilizado como critério de comparação entre os outros por apresentar, segundo a literatura, boa estimativa em relação aos constituintes da composição corporal, notadamente a água corporal total e a massa isenta de gordura e, por ser o mais empregado em ambiente laboratorial para o desenvolvimento e validação da técnica de bioimpedância ${ }^{5,6}$. Neste estudo, apesar de os resultados do RJL101 não apresentarem diferenças significativas com a pesagem e o aparelho ter mostrado o menor SEE, a correlação entre as técnicas foi fraca $(r=0,35)$ e não significativa. É importante notar, porém, que a amostra reduzida para esse aparelho aumenta os riscos de ocorrência de erros do tipo II.

Já que o enfoque deste trabalho foi testar a utilização da técnica de bioimpedância por meio de vários aparelhos, foi dada maior importância à estimativa do percentual de gordura corporal. Com isso, esperava-se obter indicações da validade dessa medida pela técnica testada. A literatura indica que as correlações entre as variáveis fornecidas pela bioimpedância (impedância, resistência e reatância) e alguns elementos da composição corporal, como água corporal total e massa isenta de gordura, são elevadas ${ }^{5,6,8,14}$. Todavia, quando se calcula o percentual de gordura a partir dessas informações, freqüentemente perde-se o poder dessa associação - de fato, as estimativas para o percentual de gordura apresentam valores de correlação mais baixos e erros de predição mais altos em comparação com as outras variáveis ${ }^{9,14,26}$. Isso vem confirmar o pressuposto principal da bioimpedância, de que não há relação teórica direta entre as variáveis da bioimpedância e a gordura percentual. A bioimpedância presta-se a estimar a água corporal 
total e, a partir daí, a massa isenta de gordura. Uma vez subtraído esse valor da massa corporal total, obtém-se a massa de gordura e, consequientemente, o seu percentual ${ }^{14}$.

Por exemplo, estudos como os de Lukaski et al. ${ }^{5}$ e o de Segal et $a{ }^{7}{ }^{7}$ identificaram erros padrões de estimativa para o \% G da ordem de $2,4 \%$ para homens a $6,1 \%$ em uma amostra combinada de homens e mulheres, enquanto Jackson et $a l .{ }^{9}$ e Lukaski et al. ${ }^{5}$ reportaram coeficientes de correlação, ainda para o \%G, entre 0,71 e 0,95. Nenhum dos aparelhos testados neste estudo mostrou índices de correlação maiores do que 0,80 e, excetuando o $R J L-101$, apresentaram altos erros padrões de estimativa. Os dados, identificando correlações reduzidas a moderadas ( $r$ variando entre 0,35 e 0,70 e o SEE entre 3,6 e 5,9) entre a bioimpedância e o percentual de gordura estimado pela pesagem hidrostática, tendem a corroborar os achados de outros estudos. Esses dados discordam dos obtidos por Lukaski et al. ${ }^{5,6}$ e Paijmans et al. ${ }^{10}$, mas confirmam os achados de Segal et al. ${ }^{7}$, Jackson et al. ${ }^{9}$, Silva ${ }^{11}$ e Carvalho e Pires Neto ${ }^{12}$. Isso aponta para um ainda não completo desenvolvimento da técnica de bioimpedância para que possamos elevá-la a um patamar de maior confiança na estimativa do percentual de gordura.

Para explicar a variabilidade nos resultados obtidos pelos diversos estudos, pode-se listar entre as fontes de erros a utilização de equações sem o real conhecimento de sua especificidade e nem de qual aparelho esta foi desenvolvida e a variação no estado de hidratação dos avalia$\operatorname{dos}^{11,14,22,26}$. Os resultados do presente estudo tendem a alinhar-se com essa opinião geral. É inegável que, ao menos a partir das informações fornecidas pelos fabricantes, não se dispõe de parâmetros para avaliar se a equação escolhida pelos aparelhos é a que se ajusta à população testada. Coincidentemente, com o único aparelho no qual houve a possibilidade de escolher a equação utilizada, o RJL-101, obteve-se o menor erro padrão de estimativa.

Quando comparamos os dados, incluindo os resultados obtidos pela técnica de dobras cutâneas, percebe-se menor variação das medidas em relação à pesagem hidrostática, $r$ $=0,85$ para o $\sum$ de três dobras e $r=0,84$ para o $\sum$ de sete dobras, enquanto o melhor índice de correlação para a bioimpedância foi da ordem de 0,7 , mas este sendo aparente em um aparelho que apresentou carência em sua precisão de medida. Já em relação aos erros padrões de estimativa, as duas equações os apresentaram menores do que qualquer um dos equipamentos de bioimpedância, indicando menor variabilidade na medida que leva a creditar, independentemente das suas limitações ${ }^{2,21,27}$, maior confiança sobre os resultados das estimativas para o percentual de gordura, pelo menos na população avaliada. Esses dados tendem a concordar com os obtidos por Jackson e Pol- $\operatorname{lock}^{15}$, quando do desenvolvimento das referidas equações, e vão ao encontro de uma opção de utilização mais consistente.

Em suma, foram detectadas diferenças significativas entre os resultados do percentual de gordura obtidos pelos aparelhos A-310 da Byodinamics e BF-906 da Maltron e a pesagem hidrostática. Isso não ocorreu quando comparados os aparelhos de bioimpedância entre si. As diferenças encontradas podem estar associadas ao erro da medida de impedância realizada pelo equipamento, ao erro na escolha da equação a ser utilizada, à falta de especificidade da equação utilizada para a amostra em questão ou à combinação de uma ou mais dessas razões.

Até o momento, não há dados que permitam indicar um aparelho em detrimento de outro. Em nossa opinião, os resultados equivalem-se quanto ao poder de estimativa do percentual de gordura, suas vantagens e desvantagens decorrendo mais do contexto da utilização. A bioimpedância continua a ser uma técnica de futuro promissor, necessitando de mais estudos, principalmente, no desenvolvimento de equações específicas, tanto para diferentes populações, quanto para a utilização em diferentes equipamentos, e na investigação de seus pressupostos básicos, com a finalidade de minimizar as suas limitações. A técnica de dobras cutâneas, conforme a metodologia/equações propostas por Jackson e Pollock ${ }^{15}$, mostra-se uma boa opção de utilização para a estimativa mais confiável da gordura corporal.

No que diz respeito às possibilidades de estudos futuros, algumas recomendações poderiam ser traçadas. Os resultados apresentados, por exemplo, devem ser ratificados pela ampliação do universo amostral, a fim de que erros do tipo II possam ser controlados na mesma proporção que os erros do tipo I. Da mesma forma, são necessários estudos controlando ao máximo as limitações apontadas, para testar a validade das equações utilizadas pelos diversos equipamentos em diferentes populações. A realização da medida direta do volume residual para a estimativa do ar presente nos pulmões durante a técnica da pesagem hidrostática e a tomada de medida da água corporal total de cada indivíduo, refinando a análise comparativa com os dados obtidos pelos aparelhos, seriam algumas das providências que poderiam ser adotadas para aumentar a validade externa do delineamento metodológico.

\section{AGRADECIMENTOS}

Agradecemos à Proximus Tecnologia Ltda. e ao Instituto de Nutrição da UFRJ, pelo apoio material ao desenvolvimento da pesquisa. Estudo parcialmente financiado pela Capes, através de seu programa de demanda social. 


\section{REFERÊNCIAS}

1. Heyward VH, Stolarczyk LM. Applied body composition assessment. Champaign: Human Kinetics, 1996.

2. Pollock ML, Wilmore JH. Exercícios na saúde e na doença. Avaliação e prescrição para prevenção e reabilitação. $2^{\underline{a}}$ ed. Rio de Janeiro: MEDSI, 1993.

3. Brodie DA. Techniques of measurement of body composition: Part I. Sports Med 1988;5:11-40.

4. Brodie DA. Techniques of measurement of body composition: Part II. Sports Med 1988;5:74-98.

5. Lukaski HC, Johnson PE, Bolonchuk WW, Lykken GI. Assessment of fat-free mass using bioelectrical impedance measurements of human body. Am J Clin Nutr 1985;41:810-7.

6. Lukaski HC, Bolonchuk WW, Hall CB, Siders WA. Validation of tetrapolar bioelectrical impedance method to assess human body composition. J Appl Physiol 1986;60:1327-32.

7. Segal KR, Gutin B, Presta E, Wang J, van Itallie TB. Estimation of human body composition by electrical impedance methods: a comparative study. J Appl Physiol 1985;58:1565-71.

8. Kushner RF, Schoeller DA. Estimation of total body water by bioelectrical impedance analysis. Am J Clin Nutr 1986;44:417-24.

9. Jackson AS, Pollock ML, Graves JE, Mahar MT. Reliability and validity of bioelectrical impedance in determining body composition. J Appl Physiol 1988;64:529-34.

10. Paijmans IJ, Wilmore KM, Wilmore JH. Use of skinfolds and bioelectrical impedance for body composition assessment after weight reduction. J Am Coll Nutr 1992;11:145-51.

11. Silva SC. Fidedignidade e objetividade da medida de impedância bioelétrica na avaliação da gordura percentual em adultos do sexo masculino. Dissertação de mestrado. Rio de Janeiro: PPGEF/UGF, 1997.

12. Carvalho A, Pires Neto C. Composição corporal através dos métodos da pesagem hidrostática e impedância bioelétrica em universitários. Revista Brasileira de Cineantropometria e Desenvolvimento Humano 1999; $1: 18-23$.

13. Kushner RF. Bioelectrical impedance analysis: a review of principles and applications. J Am Coll Nutr 1992; 11:199-209.
14. National Institutes of Health. Bioelectrical impedance analysis in body composition measurement: National Institutes of Health Technology Assessment Conference Statement. Am J Clin Nutr 1996;64:524S-32S.

15. Jackson AS, Pollock ML. Generalized equations for predicting body density of men. Br J Nutr 1978;40:497-504.

16. Siri WE. Body composition from fluid spaces and density. In: Brozek J, Henschel A, editors. Techniques for measuring body composition. Washington DC: National Academy of Science, 1961:223-44.

17. Hoffer EC, Meador CK, Simpson DC. Correlation of whole-body impedance with total body water volume. J Appl Physiol 1969;27:531-4.

18. Behnke AR, Wilmore JH. Evaluation and regulation of body build and composition. Englewoods Cliffs: Prentice-Hall, 1974.

19. Goldman HI, Becklake MR. Respiratory function tests: normal values of medium altitudes and the prediction of normal results. American Review of Tuberculosis and Respiratory Disease 1959;79:457-67.

20. Buskirk ER. Underwater weighing and body density: a review of procedures. In: Brozek J, Henschel A, editors. Techniques for measuring body composition. Washington DC: National Academy of Science, 1961:90105.

21. Guedes DP. Estudo da gordura corporal através da mensuração dos valores de densidade corporal e da espessura de dobras cutâneas em universitários. Dissertação de mestrado. Santa Maria: UFSM, 1985.

22. Graves JE, Pollock ML, Colvin A, Van Loan M, Lohman TG. Comparison of different bioelectrical impedance analyzers in the prediction of body composition. American Journal of Human Biology 1989;1:60311.

23. Zar JH. Biostatistical analysis. $4^{a}$ ed. New Jersey: Prentice Hall, 1999.

24. Wilmore J. The use of actual, predicted and constant residual volumes in assessment of body composition by underwater weighing. Med Sci Sports Exerc 1969;1:87-90.

25. Lohman T. Advances in body composition assessment. Champaign: Human Kinetics, 1992.

26. Pollock M, Garzarella L, Graves J. The measurement of body composition. In: Maud P, Foster C, editors. Physiological assessment of human fitness. Champaign: Human Kinetics, 1995:185-91.

27. Guedes DP. Composição corporal: princípios, técnicas e aplicações. $2^{2}$ ed. Londrina: APEF, 1994. 